\title{
Assessing reactive power reserves with respect to operating constraints and voltage stability
}

\author{
Florin Capitanescu
}

\begin{abstract}
This paper proposes a two-step approach to evaluate reactive power reserves with respect to operating constraints and voltage stability for a set of postulated operating scenarios. The first step determines the minimum overall needed reactive power reserves of generators such that the system withstands, from a static viewpoint, any postulated scenario. This problem is formulated as a security constrained optimal power flow (SCOPF) which includes operating constraints relative to all postulated scenarios. Particular attention is paid to the techniques aimed to reduce the large size of the SCOPF problem. The second step determines additional reserves to ensure voltage stability of scenarios for which, when modeling dynamic system behaviour, the reserves obtained by SCOPF are insufficient. These reserves are computed using a heuristic technique which relies on dynamic simulation. Numerical results on four test systems of $60,118,618$, and 1203 buses support the interest of the approach.
\end{abstract}

Index Terms-optimal power flow, reactive power reserves, security constrained optimal power flow, voltage stability

\section{INTRODUCTION}

$\mathbf{T}$ $\mathrm{HE}$ main role of reactive power reserves is to support the voltage profile in response to various disturbances and thereby to enable secure system operation. Maintaining adequate and properly located reactive power reserves is an essential condition in order to avoid: voltage instability [1][3], angle instability [1], and abnormal voltages.

A feature of reactive power is that it cannot be transmitted over long distances. For instance, even if the generator has a large reserve with respect to its physical limit, its effective ability to help for remote disturbances may be limited.

The evaluation of reactive power support and reserves has gained even more attention within the context of unbundling of generation and transmission [4]-[10]. Provision of reactive power reserve is an ancillary service that has to be valuated and paid accordingly. The value of this service must be assessed with respect to its capability of helping the system to face various operating scenarios (e.g. outages, different load levels).

A lot of work has been devoted to valuating the reactive power support [4]-[10] while comparatively less efforts have been made for evaluating reactive power reserves [10]-[19].

The reactive power reserves requirements have been mainly investigated in the context of voltage stability [10]-[18], most approaches using static models, unless [14], [15].

Ref. [10] proposes an "equivalent reactive compensation" method to valuate the reactive power support and reserves. It consists of adding fictitious synchronous condensers at

F. Capitanescu is with the Department of Electrical Engineering and Computer Science, University of Liège, B4000 Liège, Belgium (e-mail: capitane@montefiore.ulg.ac.be) selected load buses, and switching all reactive sources under constant power. The value of a generator reactive reserve is computed by a weighted sum combining the value of the reserve in various contingencies. The proper location of the synchronous condensers is a key step in order to obtain meaningful results. Ref. [11] monitors reactive power margins on pre-defined voltage control areas in order to assess the voltage profile quality. The margin is computed as the difference between the individual reserves of generators within an area and the additional reactive generation needed to maintain acceptable voltage levels after any given contingency. Ref. [12] relies on the notion of "reactive reserve basin" of an area which is defined as the sum of the reactive reserves exhausted at the minimum of the VQ curve [2], [3] relative to any bus of the area. The percentage of basin reactive reserve remaining after a disturbance is used as a measure of proximity to voltage instability. Ref. [13] proposes two methods for determining the "effective" reactive reserve of an area. The former method computes the reserve as the sum of individual reserves of the generators under limit at the minimum of the VQ curve relative to a bus or an area. The latter approach computes an effective power reserve as the weighted sum of individual reserves; the weights are based on sensitivities of generator reactive outputs to reactive loads. Ref. [14] monitors the effective reactive reserve defined as the difference between the maximum reactive power provided by generators in the marginally stable scenario relative to a contingency and their current reactive power output. The system operator is alerted as soon as, for a contingency, the effective reserve approaches the minimum reactive reserve, defined as the generators response to the contingency in the marginally stable scenario. Ref. [15] proposes a reactive reserve management scheme based on multi-objective optimal power flow in order to meet reactive power demands during voltage emergencies. The management scheme uses participation factors for each generator which are determined based on the VQ curves. Ref. [16] derives, at the nose of PV curves, constraints on reactive power reserve requirements, and include them in a SCOPF which aims to enhance voltage stability margins. Ref. [17] examines by a regression model the nonlinear relation between the reactive power reserves and both voltage stability margins and voltage limits. Ref [18] discusses several issues related to the reactive reserves as seen from both load and generation side.

Reactive power reserves have been also examined in the context of operating constraints satisfaction (e.g. voltage limits) [10], [17], [19]. Ref. [19] includes the evaluation of reactive reserves in a more general context of long-term Var sources planning. The problem is formulated as a SCOPF, in- 
cludes several pre-defined contingencies, and is solved relying on sensitivity-based linearizations of the original problem.

The main contributions of this paper are:

- a new approach to assess reactive power reserves relying on SCOPF and dynamic simulation is proposed;

- the reactive power reserves are evaluated with respect to both operating limits and voltage stability;

- techniques to reduce the size of the SCOPF are proposed.

The rest of the paper is organized as follows. Section II (resp. III) describes the first (resp. second) step of the proposed approach. Section IV presents the overall algorithm of the approach. Section V yields extensive numerical results with the approach and Section VII concludes.

\section{STEP 1: DETERMINING OPTIMAL NEEDED REACTIVE POWER RESERVES SATISFYING OPERATING CONSTRAINTS}

\section{A. Principle and assumptions}

The first step of the proposed approach computes the minimum overall needed reactive power reserve (MONRPR) of generators, and its share among them, such that the system responds (from a static viewpoint) in an acceptable way to any postulated operating scenario $^{1}$, i.e. a steady-state exists and operating constraints (e.g. limits on voltages and currents) are met. This approach is called MONRPR in the sequel.

The assessment of reactive reserves takes place after the active/reactive power dispatch has been performed [25], ensuring thereby that operating constraints are met in all scenarios.

This approach focuses on the evaluation of reserves in the reactive power production mode but can be likewise formulated for the absorption mode.

Let $\mathcal{B}, \mathcal{G}$, and $\mathcal{S}$ denote the set of: buses, generators, and postulated operating scenarios, and with $|\mathcal{B}|,|\mathcal{G}|$, and $|\mathcal{S}|$ the size of these sets, respectively.

Let scenario $s=0$ denote the base case, i.e. the system state forecasted for a given period of time of the next day (e.g. the base case may stem from the reactive power dispatch).

Let $Q_{g i}^{s}$ denote the reactive power output of generator $i$ in the scenario $s ; Q_{g i}^{s}$ satisfies the box constraints:

$$
Q_{g i}^{\min } \leq Q_{g i}^{s} \leq Q_{g i}^{\max },
$$

where $Q_{g i}^{\min }$ and $Q_{g i}^{\max }$ are physical reactive power limits ${ }^{2}$.

In the proposed approach a new variable $R_{g i}^{O C}$, independent of the operating scenario, is defined for each generator $i \in$ $\mathcal{G}$ to model its needed reactive reserve to satisfy operating constraints (OC) for all scenarios. $R_{g i}^{O C}$ obeys the constraints:

$$
0 \leq R_{g i}^{O C} \leq Q_{g i}^{\max }-Q_{g i}^{0},
$$

$Q_{g i}^{0}$ being the reactive power of generator $i$ in the base case. Taking (2) into account the constraints (1) become:

$$
Q_{g i}^{\min } \leq Q_{g i}^{s} \leq Q_{g i}^{0}+R_{g i}^{O C}
$$

\footnotetext{
${ }^{1}$ An operating scenario may include: contingencies (i.e. transmission or generation equipments outages), different load/generation dispatch, or both.

${ }^{2}$ They should reflect the limits imposed in the generator field current [3]. This work assumes constant reactive power limits $\left(Q_{g i}^{\min }\right.$ and $\left.Q_{g i}^{\max }\right)$ although they depend on generator' terminal voltage and active power.
}

The proposed approach looks for the values of reactive reserves $R_{g i}^{O C}$ 's which minimize the overall needed reactive power reserves of generators, or maximize the overall (useless part of) physical reserve withdrawn from generators:

$$
\min \sum_{i \in \mathcal{G}} R_{g i}^{O C} \text {, or } \max \sum_{i \in \mathcal{G}}\left(Q_{g i}^{\max }-Q_{g i}^{0}-R_{g i}^{O C}\right) \text {, }
$$

while satisfying operating constraints for all scenarios $s \in \mathcal{S}$.

The evaluation of reactive reserves requires properly modeling the three modes of generators operation [3], [20]:

1) under voltage control, satisfying the constraints: $Q_{g i}^{\min }<Q_{g i}^{s}<Q_{g i}^{\max }$ and $V_{g i}^{s}=V_{g i}^{i m p}$

2) in over-excitation mode, satisfying the constraints: $Q_{g i}^{s}=Q_{g i}^{\max }$ and $V_{g i}^{s} \leq V_{g i}^{i m p}$

$3)$ in under-excitation mode, satisfying the constraints: $Q_{g i}^{s}=Q_{g i}^{\min }$ and $V_{g i}^{s} \geq V_{g i}^{i m p}$.

A drawback of the classical SCOPF is that it does not model the switch between generator modes, which would dramatically affect the robustness of nonlinear programming solvers, the generator being modeled by constraints (1) and assuming that its voltage is maintained at an imposed value:

$$
V_{g i}^{s}=V_{g i}^{i m p}, i \in \mathcal{G}, s \in \mathcal{S} \text {. }
$$

Note that if a classical SCOPF program is used to optimize the objective (4) and includes, beside typical operating constraints, the constraints (2), (3), and (5), then at the solution the reactive reserve of each generator is merely the maximum reactive power response of the generator over all scenarios.

Clearly, the same reactive reserves ${ }^{3}$ can be obtained in a much simpler way i.e. by merely simulating all postulated scenarios by a power flow program and taking the maximum reactive power response (MRPR) of each generator over all scenarios. This approach is called MRPR in the sequel.

An appealing solution consists in handling in the SCOPF the generator status switch between these modes by complementarity constraints [20], transforming the problem into a mathematical program with equilibrium constraints (MPEC).

The complementarity constraints modeling the behaviour of generator $i$ in any scenario $s \in \mathcal{S}$ can be expressed as ${ }^{4}$ :

$$
0 \leq\left(Q_{g i}^{0}+R_{g i}^{O C}-Q_{g i}^{s}\right) \perp\left(V_{g i}^{i m p}-V_{g i}^{s}\right) \geq 0,
$$

the operator $\perp$ denoting the complementarity of two quantities.

Note that, in order to separate needed and useless generator reserves, constraint (6) enables the generator $i$ to enter in overexcitation mode for any value of the reserve $R_{g i}^{O C}$, and hence possibly before the generator reaches its physical reactive power limit, provided that the objective (4) is improved.

MPEC solvers behave well for OPF problems modeling a small number of complementarity constraints [20] but encounter reliability problems as the number of complementarity constraints grows [21]. Hence the reliable handling of a large number of complementarity constraints, as required by the

\footnotetext{
${ }^{3}$ Provided that no generator reaches its reactive power limit switching thereby its status from a PV bus to a PQ bus.

${ }^{4} \mathrm{As}$ the focus is on the reactive reserves in production mode the complementarity constraints modeling the under-excitation mode are neglected. This is a fair assumption because operating scenarios are generally severe and hence most generators react by producing more reactive power.
} 
proposed approach in real-life systems ${ }^{5}$, has to be proven. For these reasons OPF problems with complementarity constraints are genrally tackled by heuristics [9].

In the proposed approach the complementarity constraints (6) are replaced with the following weaker constraints that capture the generator behaviour in over-excitation mode:

$$
\begin{array}{r}
Q_{g i}^{s} \leq Q_{g i}^{0}+R_{g i}^{O C} \\
V_{g i}^{s} \leq V_{g i}^{i m p},
\end{array}
$$

where $R_{g i}^{O C}$ obeys constraints (2).

Note that, although constraints (7)-(8) do not capture the generator behaviour under voltage control (since if $Q_{g i}^{s}<$ $Q_{g i}^{0}+R_{g i}^{O C}$ holds does not guarantee that the free variable $V_{g i}^{s}$ tends to $V_{g i}^{i m p}$ ), fortunately this generator mode is naturally met $^{6}$ at optimum. This is due to the larger the generator voltage $V_{g i}^{s}$, the larger the generator reactive power output, the smaller the reactive power losses, the smaller the generators reactive power response in a scenario and hence the smaller the needed reserves. Extensive tests with the proposed SCOPF confirm this observation, further details being provided in section V-E.

\section{B. MONRPR approach formulation as a SCOPF}

The SCOPF model of the approach is described hereafter.

1) Objective function: minimize the overall needed reactive power reserves of generators:

$$
\min _{\mathbf{R}_{g}^{O C}, \mathbf{V}_{g}^{s}} \sum_{i \in \mathcal{G}} R_{g i}^{O C} .
$$

2) Control variables: the needed reactive reserves of generators $R_{g i}^{O C}, i \in \mathcal{G}$ and the generators voltages $V_{g i}^{s}, i \in \mathcal{G}, s \in$ $\mathcal{S} \backslash\{0\}$ for all postulated scenarios unless the base case.

3) Constraints: Equality constraints encompass the active and reactive power flow equations, written for each bus $i \in \mathcal{B}$ and for each postulated operating scenario $s \in \mathcal{S}$ :

$$
\begin{array}{r}
P_{g i}^{s}-P_{l i}^{s}-\sum_{j \in \mathcal{B}_{i}^{s}} P_{i j}^{s}\left(V_{i}^{s}, V_{j}^{s}, \theta_{i}^{s}, \theta_{j}^{s}\right)=0 \\
Q_{g i}^{s}-Q_{l i}^{s}-\sum_{j \in \mathcal{B}_{i}^{s}} Q_{i j}^{s}\left(V_{i}^{s}, V_{j}^{s}, \theta_{i}^{s}, \theta_{j}^{s}\right)=0,
\end{array}
$$

where, in scenario $s, P_{g i}^{s}$ (resp. $Q_{g i}^{s}$ ) is the active (resp. reactive) power generated at bus $i, P_{l i}^{s}$ (resp. $Q_{l i}^{s}$ ) is the active (resp. reactive) load at bus $i, P_{i j}^{s}$ (resp. $Q_{i j}^{s}$ ) is the active (resp. reactive) power flow between buses $i$ and $j, \mathcal{B}_{i}^{s}$ is the set of buses directly connected to bus $i$, and $V_{i}^{s}$ (resp. $\theta_{i}^{s}$ ) is the voltage magnitude (resp. angle) at bus $i$.

Generator terminal voltages are imposed ${ }^{7}$ in the base case (e.g. at values agreed with the TSO and/or at optimal values stemming from reactive power dispatch):

$$
V_{g i}^{0}=V_{g i}^{i m p} \text {. }
$$

\footnotetext{
${ }^{5}$ The adequate modeling of generator voltage control mechanism leads to include $2 \times|\mathcal{G}| \times|\mathcal{S}|$ complementarity constraints in the SCOPF.

${ }^{6}$ Incidentally this behaviour also appears when computing loadability margins by OPF if complementarity constraints $0 \leq\left(Q_{g i}^{\max }-Q_{g i}^{s}\right) \perp$ $\left(V_{g i}^{i m p}-V_{g i}^{s}\right) \geq 0$ [20] are replaced by the constraints (1) and (8).

${ }^{7}$ Due to the nature of the objective, even if voltages are modeled as $V_{g i}^{0} \leq$ $V_{g i}^{i m p}$, the SCOPF provided the same solution in all numerical tests.
}

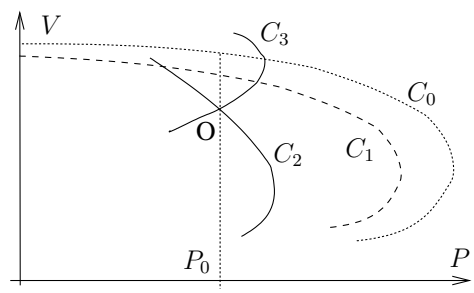

Fig. 1. Intuitive view of the SCOPF solution for a binding scenario.

Inequality constraints in each scenario $s \in \mathcal{S}$ include: operational limits on branch currents $\left(i \in \mathcal{B}, j \in \mathcal{B}_{i}^{s}\right)$ :

$$
I_{i j}^{s}\left(V_{i}^{s}, V_{j}^{s}, \theta_{i}^{s}, \theta_{j}^{s}\right) \leq I_{i j}^{\max },
$$

voltage magnitude limits at non-generator bus $i \in \mathcal{B} \backslash \mathcal{G}$ :

$$
V_{i}^{\min } \leq V_{i}^{s} \leq V_{i}^{\max },
$$

voltage magnitude limits at generator $i \in \mathcal{G}, s \in \mathcal{S} \backslash\{0\}$ :

$$
V_{i}^{\text {min }} \leq V_{g i}^{s} \leq V_{g i}^{i m p},
$$

allowing generators to enter in over-excitation mode enabling the optimization process to identify useless physical reserves; active/reactive power limits of generator $i \in \mathcal{G}$ :

$$
\begin{array}{r}
P_{g i}^{\min } \leq P_{g i}^{s} \leq P_{g i}^{\max } \\
Q_{g i}^{\min } \leq Q_{g i}^{s} \leq Q_{g i}^{0}+R_{g i}^{O C},
\end{array}
$$

and limits on the needed reactive reserve of generator $i \in \mathcal{G}$ :

$$
0 \leq R_{g i}^{O C} \leq Q_{g i}^{\max }-Q_{g i}^{0} .
$$

\section{Remarks}

Although the approach focuses on technical aspects of reactive reserves it can be easily extended to a market environment, e.g. by considering bidding costs for reserves $R_{g i}^{O C}$ 's in (9) [9].

The approach can easily incorporate mandatory reactive power ranges (e.g. 0.95 lead to 0.95 lag power factor) [9].

Clearly, due to the control variables employed and that it is performed after the active/reactive power dispatch, the approach is not suitable to restore a power flow solution or to remove violated limits in a scenario.

Slower reactive power controls (e.g. shunt devices and transformers taps) are not considered in optimization since they are generally set in the reactive power dispatch.

\section{Relation between the SCOPF solution and voltage stability}

Figure 1 provides an intuitive view by means of PV curves of the solution obtained by the proposed SCOPF for any binding scenario, neglecting voltage limits. The PV curve $C_{0}$ (resp. $C_{1}$ ) corresponds to the system operation in base case (resp. chosen scenario) where the whole system physical reactive reserves are available. The proposed procedure withdraws as much reactive reserve as possible, shrinking hence the PV curve, as long as it still intersects the constant power load characteristic $P_{0}$. While "cutting" reactive reserves, generators are allowed to switch to the over-excitation mode if this improves the objective. Consequently, generators 
voltages drop, as shown by curve $C_{2}$. The procedure eventually converges to the optimum, the point $\mathrm{O}$, which corresponds to the intersection between the load characteristic $P_{0}$ and the PV curves $C_{2}$ and $C_{3}$, where curve $C_{3}$ corresponds to generators that switch to over-excitation mode but their voltages remain at the imposed value, i.e. both inequalities (7) and (8) are active.

The limit point $\mathrm{O}$ is referred to as a breaking point (or limit induced bifurcation) and is a voltage stability limit (see Chapter 7 pp. 258-259 [3]), further evidence being provided in Section V-E. Therefore the SCOPF solution satisfies scenarios voltage stability assuming a static constant power load model.

\section{STEP 2: DETERMINING ADDITIONAL RESERVES TO ENSURE VOLTAGE STABILITY}

If dynamic aspects are taken into account the optimal reserves $\mathbf{R}_{g}^{O C \star}=\left[R_{g 1}^{O C \star}, \ldots, R_{g i}^{O C \star}, \ldots, R_{g|\mathcal{G}|}^{O C \star}\right]^{T}$ provided by SCOPF may not suffice to ensure scenarios voltage stability.

Since the nonlinear behaviour of reactive power flows in normal operation is much more pronounced and discontinuous in voltage unstable or marginally stable scenarios computing in a reasonable time the optimal additional reserves to ensure scenarios voltage stability is very challenging if not utopian.

The benchmark solution, the Monte Carlo simulation (i.e. generate all possible combinations of generators reactive reserves, simulate all unstable scenarios for each such combination, and taking as optimum the combination with the least overall needed reserves which ensures scenarios voltage stability), is extremely computationally demanding and hence infeasible in the time allowed to evaluate reactive reserves.

Therefore, one has to rely on faster heuristic techniques which look for a reasonable (hopefully sub-optimal) solution.

The proposed iterative procedure relies on two ideas:

- At each iteration only the reactive reserve requirements for the worst scenario are satisfied. As a consequence, the remaining unstable scenarios benefit of larger reserves and hence may need smaller additional reserves.

- For a scenario the minimum overall needed reserves corresponds to the reactive response of the generators (considering their entire physical reserves).

Let $\mathcal{U}$ denote the subset of voltage unstable scenarios found by dynamic simulation for the SCOPF-based reserves $\mathbf{R}_{g}^{O C \star}$.

The algorithm of this procedure applies if $\mathcal{U} \neq \emptyset$ as follows:

1) Initialize the needed reserves to ensure voltage stability $\mathbf{R}_{g}^{V S} \leftarrow \mathbf{0}$

2) Simulate scenarios from subset $\mathcal{U}$ by dynamic simulation for the current generators reserves $\mathbf{R}_{g}^{O C \star}+\mathbf{R}_{g}^{V S}$.

- If no scenario leads to voltage instability then $\mathbf{R}_{g}^{V S}$ are the sought reserves and computation terminates.

3) Rank unstable scenarios in decreasing order of a severity measure (e.g. the minimum unrestored load, i.e. the smallest difference between the base case total load and the total load recorded during the unstable trajectory).

4) Re-simulate the most severe scenario using the physical reactive power reserves of all generators.

- Let $Q_{g i}^{s D S}$ denote the reactive output of generator $i$ in the dynamic simulation of the stable scenario $s$.
- If the response of generator $i$ in scenario $s$ is larger than its current reserve, i.e. $Q_{g i}^{s D S}>Q_{g i}^{0}+R_{g i}^{O C \star}+R_{g i}^{V S}$, then update the needed reserve for voltage stability: $R_{g i}^{V S} \leftarrow Q_{g i}^{s D S}-Q_{g i}^{0}-R_{g i}^{O C \star}$.

- Go to step 2.

\section{OVERALL ALGORITHM OF THE PROPOSED APPROACH}

\section{A. Motivation and description of the overall algorithm}

The major challenge of the proposed approach is that the SCOPF problem (9)-(18) has an onerous size for large systems and/or a large number of scenarios [25]. Because most postulated scenarios do not constrain the optimum the SCOPF is solved by an iterative algorithm which includes in the SCOPF at each iteration only a subset of potentially binding 8 scenarios, denoted by $\mathcal{S}_{b}$. Clearly, the quick and reliable identification of binding scenarios is essential in order to speed up computations. Most approaches add new potentially binding scenarios to the existing ones in set $\mathcal{S}_{b}$ as iterations progress [22], [25]. However, this may lead to a non-negligible size of set $\mathcal{S}_{b}$ and hence a very large size SCOPF problem. In this work, an additional rule is used to preserve a more tractable SCOPF size namely scenarios from $\mathcal{S}_{b}$ that have not been binding at any SCOPF solution during algorithm iterations are discarded, taking the potential risk to perform new loops if those scenarios become binding at next iterations.

The proposed algorithm contains the following steps ${ }^{9}$ :

\section{1) Initialization}

Assume a given operating point and a set of postulated scenarios $\mathcal{S}$ with respect to which generators reactive reserves are evaluated.

Save the original generators reactive power limits $Q_{g i}^{\max 0} \leftarrow Q_{g i}^{\max }, i \in \mathcal{G}$.

Let $\mathcal{S}_{b} \leftarrow \emptyset$.

2) Security analysis (SA)

Simulate by a power flow with optimal multiplier (PFOM) program (see section IV-B) each scenario $s \in \mathcal{S} \backslash \mathcal{S}_{b}$, taking into account the current values of generators reactive power limits $Q_{g i}^{\max }, i \in \mathcal{G}$.

3) Termination criterion for SCOPF-based reserves If $\mathcal{S}_{b}=\emptyset$ go to the next step.

If for the current values of reactive reserves any scenario $s \in \mathcal{S}$ has a power flow solution and operating constraints are met, then $\mathbf{R}_{g}^{O C \star}$ are the optimal values of the reactive reserves satisfying operational constraints; go to step 6.

4) Scenarios filtering $(S F)$

Select a subset $\mathcal{S}_{s} \subset \mathcal{S} \backslash \mathcal{S}_{b}$ of additional potentially binding scenarios by using a three-layer scenarios filtering technique (see section IV-C). Let $\mathcal{S}_{b} \leftarrow \mathcal{S}_{b} \cup \mathcal{S}_{s}$;

5) $\mathrm{SCOPF}$

\footnotetext{
${ }^{8}$ The set of the binding scenarios at the SCOPF optimum is the smallest subset of the set $\mathcal{S}$ which provides the same optimum as the set $\mathcal{S}$.

${ }^{9}$ The part of the algorithm related to the SCOPF differs from [22] by techniques used for scenarios filtering and security analysis.
} 
- Reset generators reactive power limits to their original values $Q_{g i}^{\max } \leftarrow Q_{g i}^{\max 0}, i \in \mathcal{G}$.

- Solve the SCOPF (9)-(18) by including, besides base case constraints, only the constraints relative to the scenarios for the subset $\mathcal{S}_{b}$.

- Let $\mathbf{R}_{g}^{O C \star}=\left[R_{g 1}^{O C \star}, \ldots, R_{g i}^{O C \star}, \ldots, R_{g|\mathcal{G}|}^{O C \star}\right]^{T} \mathrm{de}-$ note the optimal values of the needed reactive reserves provided by the SCOPF.

- Update the reactive power limits of generators: $Q_{g i}^{\max } \leftarrow Q_{g i}^{0}+R_{g i}^{O C \star}, i \in \mathcal{G}$.

- Remove form the set $\mathcal{S}_{b}$ those scenarios that have never been binding at any iteration of the algorithm. Go to step 2.

6) Check reserves by dynamic simulation

Check by dynamic simulation whether the SCOPFbased reserves $\mathbf{R}_{g}^{O C \star}$ ensure the voltage stability of all postulated scenarios.

If all scenarios are voltage stable, $\mathbf{R}_{g}^{O C \star}$ are the sought optimal reserves and computation terminate. Otherwise let subset $\mathcal{U}$ denote the voltage unstable scenarios.

7) Compute additional reserves to ensure voltage stability Apply the procedure described in Section III to determine the additional reactive reserves $\mathbf{R}_{g}^{V S}$ required to ensure voltage stability of scenarios from subset $\mathcal{U}$. $\mathbf{R}_{g}^{O C \star}+\mathbf{R}_{g}^{V S}$ are the sought reactive reserves and computations terminate.

This approach proposes to reward each generator $i$ only for the reactive reserve $R_{g i}^{O C \star}+R_{g i}^{V S}$.

Observe that the $\mathrm{SA}$ uses the reactive reserves computed by the SCOPF, while the latter uses the physical reactive reserves.

\section{B. Security analysis by power flow with optimal multiplier}

Note that the reactive reserves $\mathbf{R}_{g}^{O C \star}$ at an iteration of the SCOPF algorithm may lead to cases where, for some scenarios, there is no power flow solution or the system is close to the loadability limit. Using power flow divergence as static voltage instability criterion does not allow distinguishing between these cases because divergence may result from purely numerical problems (e.g. when many generators switch under reactive power limit). To avoid these drawbacks as well as to enable the rank of scenarios which have no power flow solution the PFOM ${ }^{10}$ approach [23] is used.

\section{Scenarios filtering}

The algorithm uses sequentially three filters which exploits the information provided by the SA. To address the particular features of this SCOPF problem new filters, namely the first and the third, are required compared to those proposed in [22].

The first filter selects all scenarios $\mathcal{S}_{s}^{1} \subset \mathcal{S} \backslash \mathcal{S}_{b}$ that do not have a power flow solution as the most dangerous ones. These are ranked according to the severity index [24]:

$$
S I_{1}(s)=\sum_{i \in \mathcal{B}}\left[\left(\Delta P_{i}^{s}\right)^{2}+\left(\Delta Q_{i}^{s}\right)^{2}\right],
$$

\footnotetext{
${ }^{10}$ In this approach the step-length taken in the Newton direction is adjusted according to the optimal value of a scalar multiplier which minimizes the sum of the square mismatches of the power flow equations. In particular the optimal multiplier tends to zero and prevents computations divergence when the power flow equations have no solution.
}

TABLE I

TEST SYSTEMS CHARACTERISTICS

\begin{tabular}{|c||c|c|c|c|c|c|}
\hline system & $|\mathcal{B}|$ & $|\mathcal{G}|$ & $|\mathcal{C}|$ & $|\mathcal{L}|$ & $|\mathcal{T}|$ & $|\mathcal{R}|$ \\
\hline \hline Nordic32 & 60 & 23 & 22 & 57 & 31 & 12 \\
\hline IEEE118 & 118 & 54 & 91 & 175 & 11 & 14 \\
\hline 618-bus & 618 & 72 & 352 & 810 & 247 & 25 \\
\hline 1203-bus & 1203 & 177 & 767 & 1394 & 403 & 36 \\
\hline
\end{tabular}

where $\Delta P_{i}^{s}$ and $\Delta Q_{i}^{s}$ are the mismatches of power flow equations (10)-(11) at bus $i$ provided by the PFOM tool [24].

The second filter pre-selects the scenarios $\mathcal{S}_{s}^{2} \subset \mathcal{S} \backslash \mathcal{S}_{b} \backslash \mathcal{S}_{s}^{1}$ that lead to operational constraints violation according to the constraints non-domination [22]. It ranks the scenarios from $\mathcal{S}_{s}^{2}$ according to the overall constraints violation [22], [25]:

$$
S I_{2}(s)=\sum_{i \in \mathcal{B}} \max \left\{0, V_{i}^{\min }-V_{i}^{s}, V_{i}^{s}-V_{i}^{\max }\right\} .
$$

The third filter pre-selects the scenarios $\mathcal{S}_{s}^{3} \subset \mathcal{S} \backslash \mathcal{S}_{b} \backslash \mathcal{S}_{s}^{1} \backslash$ $\mathcal{S}_{S}^{2}$ that lead to the largest reactive power response for each generator, i.e. the smallest subset $\mathcal{S}_{s}^{3}$ that satisfies the property:

$$
\begin{aligned}
& \forall i \in \mathcal{G}, \exists s \in \mathcal{S}_{s}^{3} \text { such that: } \\
& \forall s^{\prime} \in \mathcal{S} \backslash \mathcal{S}_{b} \backslash \mathcal{S}_{s}^{1} \backslash \mathcal{S}_{s}^{2} \backslash \mathcal{S}_{s}^{3}, Q_{g i}^{s}-Q_{g i}^{0}>Q_{g i}^{s^{\prime}}-Q_{g i}^{0} .
\end{aligned}
$$

It ranks the scenarios from $\mathcal{S}_{s}^{3}$ according to the generators overall reactive power response in each scenario:

$$
S I_{3}(s)=\sum_{i \in \mathcal{G}} \max \left\{0, Q_{g i}^{s}-Q_{g i}^{0}\right\} .
$$

1) Remarks: The final ranking of scenarios is performed using the following order of subsets: $\mathcal{S}_{s}^{1}, \mathcal{S}_{s}^{2}$, and $\mathcal{S}_{s}^{3}$.

The intuitive idea of these $S I \mathrm{~s}$ is that the larger the index for a scenario the higher the chances that the scenario is binding.

Note that at the first iteration of the SCOPF algorithm only the third filter is activated since the starting point satisfies operational constraints for all scenarios while at the subsequent iterations the first filter has the most important role since it is expected that many scenarios have no power flow solution.

The filtering scheme may use an additional rule that selects only a desired number of top ranked scenarios.

The filtering scheme may take advantage of any pre-defined reactive power areas in the system, e.g. by performing a separate scenarios filtering in each area.

\section{NUMERICAL RESULTS}

\section{A. Description of the test systems}

This section presents results obtained with the proposed approach on four test systems: a 60-bus modified variant of the Nordic32 system (see Fig. 2) [28], the IEEE118 system [29], and two modified old planning models of the RTE (the French TSO) system of respectively 618 and 1203 buses.

Table I provides the main characteristics of these systems, $|\mathcal{B}|,|\mathcal{G}|,|\mathcal{C}|,|\mathcal{L}|,|\mathcal{T}|$, and $|\mathcal{R}|$ denoting respectively the number of buses, generators, loads, lines, transformers, and shunts. 


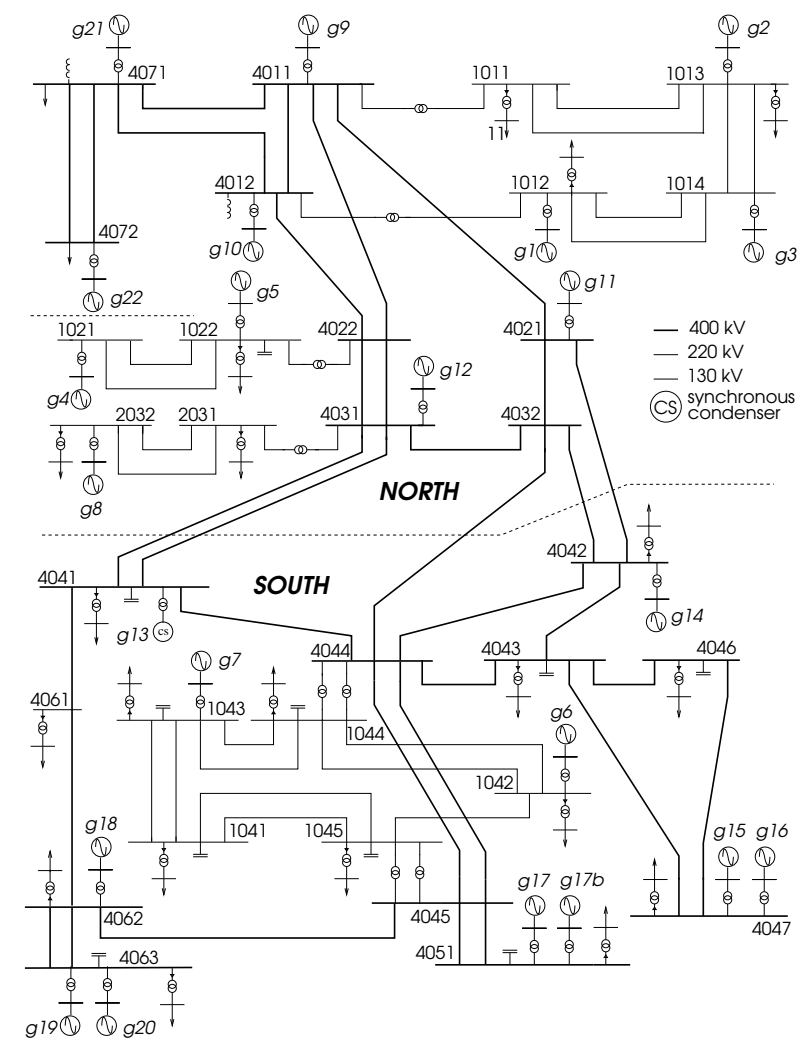

Fig. 2. The modified Nordic32 test system.

\section{B. Illustrative examples of MONRPR approach benefit}

An illustrative comparison between MONRPR and MRPR approaches for different types of contingencies is conducted on the Nordic32 system (see Fig. 2).

Note that, as expected, MONRPR and MRPR approaches provide the same results for a single scenario, i.e. the SCOPFbased optimal reactive reserves coincide with the generators response to the scenario obtained with a power flow program.

1) Contingencies located far away from each other: the loss of line 4011-4071 and the loss of line 4045-4062.

The first plot of Fig. 3 displays the generators reactive power response for each contingency. Clearly, mostly Northern (resp. Southern) generators react to contingency 4011-4071 (resp. 4045-4062). Contingency 4011-4071 (resp. 4045-4062) needs 375 (resp. 356) MVar of reserve located on proper generators.

The MRPR approach requires 669 MVar and the MONRPR approach needs 655 MVar, i.e. 14 MVar less. Obviously, MRPR approach leads to conservative reactive reserves since it disregards that a large reserve imposed by a (severe) scenario on a generator can help some generators to maintain less reserves in other scenarios. However, as expected, due to the large electrical distance between the areas impacted by these contingencies, the gain of the MONRPR approach is small.

2) Contingencies located relatively close from each other: the loss of line 4046-4047 and the loss of line 4062-4063.

The second plot of Fig. 3 shows generators reactive power response for each contingency. Contingency 4046-4047 has a wider impact and requires 663 MVar while contingency $4062-$ 4063 solicits mostly three generators and requires 103 MVar.
TABLE II

OVERALL GENERATORS REACTIVE POWER RESERVES

\begin{tabular}{|c||c|c|c||c|}
\hline \multicolumn{1}{|c||}{ system } & $|\mathcal{S}|$ & \multicolumn{2}{c||c}{ case A } & case B \\
& & MONRPR & $\begin{array}{c}\text { MRPR } \\
\text { (MVar) }\end{array}$ & $\begin{array}{c}\text { MONRPR } \\
\text { (MVar) }\end{array}$ \\
\hline \hline Nordic32 & 33 & 1394 & 1764 & 1428 \\
\hline IEEE118 & 164 & 381 & 1526 & 495 \\
\hline 618-bus & 732 & 1787 & 3346 & 1805 \\
\hline 1203-bus & 876 & 1847 & 3074 & 1847 \\
\hline
\end{tabular}

The MRPR approach needs a reserve of 726 MVar while the MONRPR approach finds that the reserve required by contingency 4046-4047 suffices to meet also the constraints relative to contingency 4062-4063, although generators g19 and g20 respond significantly more for contingency 4062-4063 than for contingency 4046-4047. The MONRPR approach leads to a significant gain (e.g. 63 MVar) of overall reactive reserve compared to the MRPR approach.

\section{Comparison between MONRPR and MRPR approaches}

Table II yields the results obtained with both approaches on four systems, where $|\mathcal{S}|$ is the number of postulated scenarios.

The program used to solve the SCOPF nonlinear programming problems relies on the interior point method, Ref. [27] providing a detailed description of the model and algorithm.

Reactive power reserves are evaluated in two cases:

- case A: with respect to voltage stability, adopting a static viewpoint (see Fig. 1), by relaxing in the SCOPF the voltage limits (14);

- case $B$ : with respect to both voltage stability and operational limits (e.g. voltages and currents). Clearly, when operating limits are more constraining than voltage stability the MRPR approach can not be used.

Note that the MONRPR approach leads for all systems to a very significant reduction of the overall needed reactive reserve compared to MRPR approach, which clearly proves the interest of the approach. As expected, the MONRPR approach requires larger overall reactive reserves in case $\mathrm{B}$ than in case A, due to the shrink of the feasible region of the problem.

Fig. 4 shows the required reactive power reserve of each generator obtained with both approaches in the Nordic32 system. The main gain of reactive reserves in the MONRPR approach is obtained for the Northern generators, where the MRPR approach allocates much larger reserves than required.

Fig. 5 indicates that, since the MONRPR approach "cuts" the useless parts of generators physical reserves, for a binding scenario at the SCOPF optimum the bus voltages are lower, but still in the range, for the minimum needed reserves than for the physical reserves of generators; voltages coincide only for generators under voltage control in both cases.

\section{Illustration of the MONRPR approach algorithm}

Table III provides the size of various sets of scenarios during SCOPF algorithm iterations in the case A. In this table $\mathcal{S}_{b}^{\star}$ denotes the set of binding scenarios at the SCOPF solution. In all tests the number of selected top ranked scenarios to be included in the SCOPF has been set to $\left|\mathcal{S}_{s}\right|=5$. 

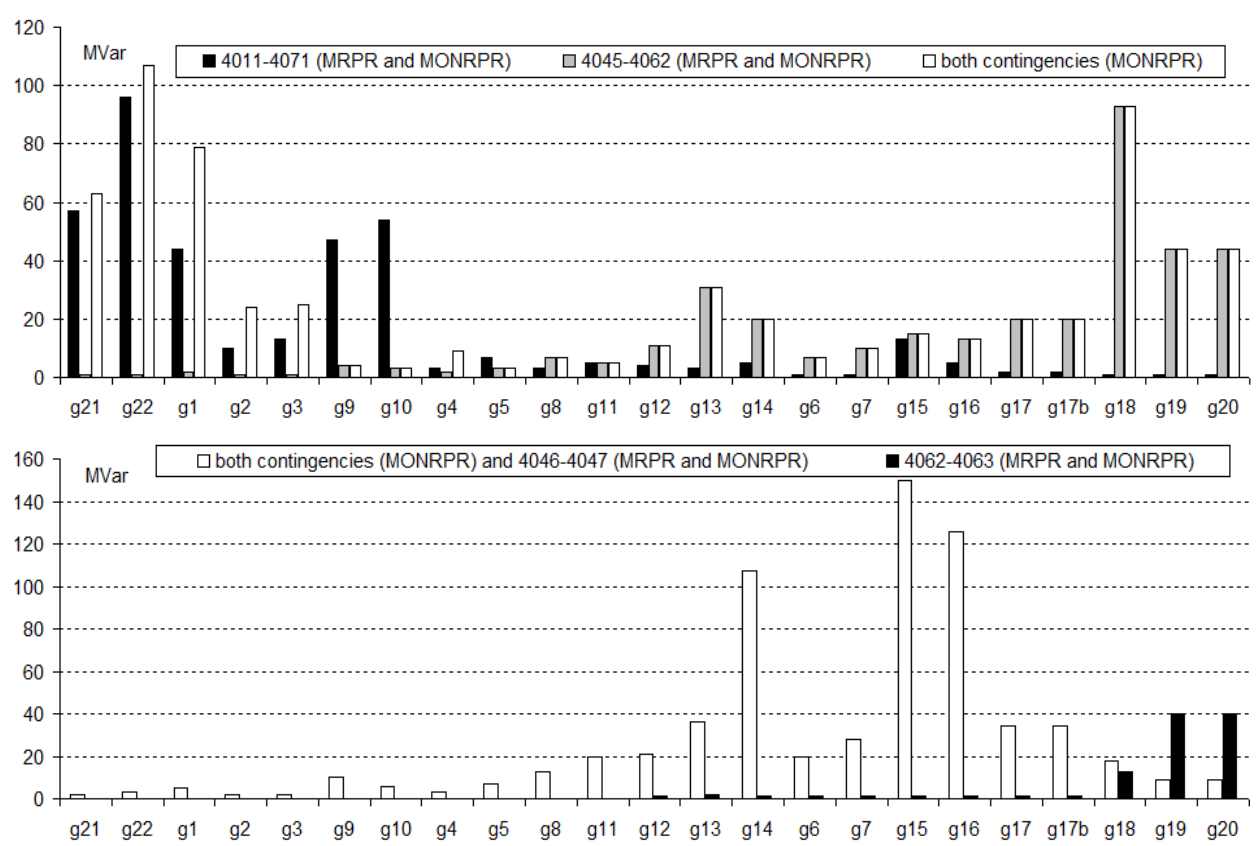

Fig. 3. Needed reactive power reserves by MONRPR and MRPR approaches for two types of contingencies.

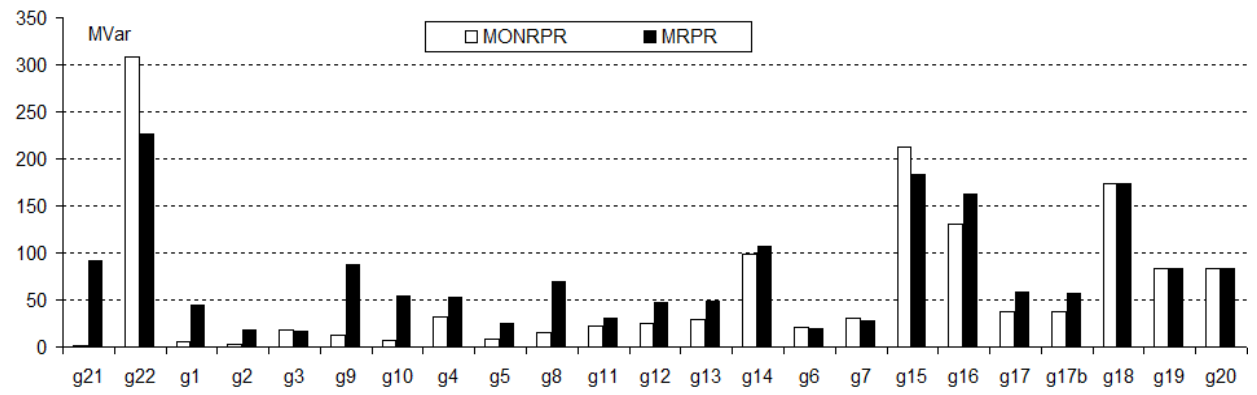

Fig. 4. Nordic32 system in case A: needed reactive reserves of generators obtained by MONRPR and MRPR approaches.

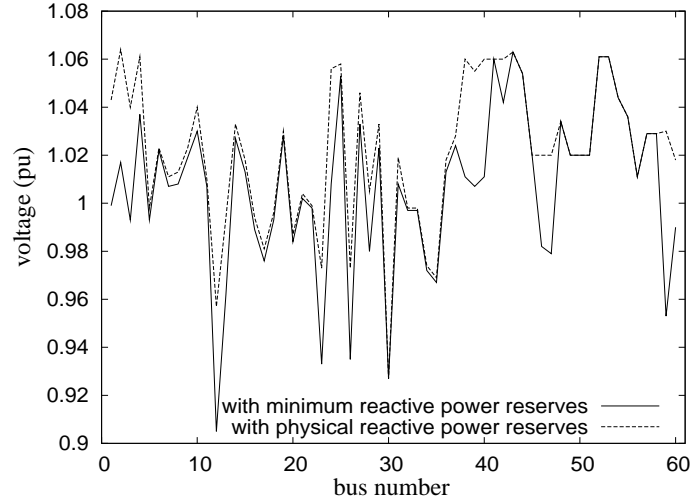

Fig. 5. Nordic32 system: bus voltages (pu) for a binding scenario in two cases: minimum reserves and physical reserves.

By looking at the results of Table III one can remark that:

- the number of loops of the algorithm is small (e.g. 2 iterations suffice to identify the binding scenarios at the optimum) thanks to the filtering techniques used;

- as iterations progress more binding scenarios are identified and the objective function increases;
TABLE III

MONRPR APPROACH IN CASE A: SIZE OF THE VARIOUS SETS OF SCENARIOS AT SUCCESSIVE ITERATIONS OF THE SCOPF ALGORITHM

\begin{tabular}{|c|c|c|c|c|c|c|c|c|}
\hline iter & $\left|\mathcal{S} \backslash \mathcal{S}_{b}\right|$ & $\left|\mathcal{S}_{s}^{1}\right|$ & $\left|\mathcal{S}_{s}^{3}\right|$ & $\left|\mathcal{S}_{s}\right|$ & $\left|\mathcal{S}_{b}\right|$ & $\left|\mathcal{S}_{b}^{\star}\right|$ & obj (MVAr) & time (s) \\
\hline \hline \multicolumn{8}{|c|}{ Nordic32 system } \\
\hline \hline 1 & 33 & 0 & 14 & 5 & 5 & 5 & 1381 & 1.2 \\
\hline 2 & 28 & 1 & 10 & 5 & 10 & 6 & 1394 & 1.3 \\
\hline 3 & 27 & 0 & - & - & - & - & - & 0.3 \\
\hline \hline \multicolumn{8}{|c|}{ IEEE118 system } \\
\hline \hline 1 & 164 & 0 & 45 & 5 & 5 & 3 & 342 & 7.5 \\
\hline 2 & 161 & 14 & 20 & 5 & 8 & 5 & 381 & 10.3 \\
\hline 3 & 159 & 0 & - & - & - & - & - & 3.2 \\
\hline \hline \multicolumn{8}{|c|}{618 -bus system } \\
\hline \hline 1 & 732 & 0 & 41 & 5 & 5 & 5 & 1212 & 57.0 \\
\hline 2 & 727 & 27 & 30 & 5 & 10 & 7 & 1787 & 69.5 \\
\hline 3 & 725 & 0 & - & - & - & - & - & 45.3 \\
\hline \hline \multicolumn{8}{|c|}{1203 -bus system } \\
\hline \hline 1 & 876 & 0 & 46 & 5 & 5 & 5 & 1815 & 173.9 \\
\hline 2 & 871 & 5 & 30 & 5 & 5 & 6 & 1847 & 238.9 \\
\hline 3 & 870 & 0 & - & - & - & - & - & 130.5 \\
\hline
\end{tabular}

- the number of binding scenarios ranges from 5 to 7 and hence does not depend on the system size or the number of postulated scenarios; 
TABLE IV

RANK OF BINDING SCENARIOS AT THE SCOPF OPTIMUM BY TWO FILTERS

\begin{tabular}{|c|c|c|c|c|c|}
\hline \multirow{2}{*}{$\begin{array}{c}\text { number of } \\
\text { binding } \\
\text { scenario }\end{array}$} & \multicolumn{3}{|c|}{ third filter } & \multicolumn{2}{|c|}{ first filter } \\
\hline & $\begin{array}{l}\text { rank without } \\
\text { pre-selection }\end{array}$ & $\begin{array}{c}\text { rank with } \\
\text { pre-selection }\end{array}$ & $\begin{array}{c}S_{3} \\
\text { (MVar) }\end{array}$ & rank & $\begin{array}{c}S I_{1} \\
\text { (MVA) }\end{array}$ \\
\hline \multicolumn{6}{|c|}{ Nordic32 system } \\
\hline 30 & 1 & 1 & 663 & - & - \\
\hline 26 & 2 & 2 & 626 & - & - \\
\hline 21 & 3 & 3 & 587 & - & - \\
\hline 30 & 4 & 4 & 424 & - & - \\
\hline 14 & 5 & 5 & 375 & - & - \\
\hline 4 & 22 & 12 & 104 & 1 & 48 \\
\hline \multicolumn{6}{|c|}{ IEEE118 system } \\
\hline 31 & 1 & 1 & 257 & - & - \\
\hline 86 & 2 & 2 & 246 & - & - \\
\hline 87 & 3 & 3 & 140 & - & - \\
\hline 125 & 12 & 11 & 70 & 1 & 21 \\
\hline 147 & 24 & 20 & 49 & 3 & 14 \\
\hline \multicolumn{6}{|c|}{ 618-bus system } \\
\hline 207 & 1 & 1 & 606 & - & - \\
\hline 695 & 4 & 3 & 478 & - & - \\
\hline 28 & 5 & 4 & 470 & - & - \\
\hline 730 & 7 & 5 & 403 & - & - \\
\hline 514 & 10 & 8 & 347 & 1 & 92 \\
\hline 680 & 21 & 15 & 279 & 5 & 70 \\
\hline 677 & 42 & 18 & 209 & 2 & 84 \\
\hline \multicolumn{6}{|c|}{ 1203-bus system } \\
\hline 276 & 1 & 1 & 1048 & - & - \\
\hline 404 & 2 & 2 & 740 & - & - \\
\hline 284 & 3 & 3 & 587 & - & - \\
\hline 283 & 4 & 4 & 584 & - & - \\
\hline 155 & 5 & 5 & 546 & - & - \\
\hline 388 & 29 & 16 & 316 & 1 & 21 \\
\hline
\end{tabular}

Table IV provides for two filters the rank and the value of severity index of the binding scenarios at the SCOPF optimum.

1) Third filter pre-selection performance: It was noticed that binding scenarios always lead to the maximum reactive power response over all scenarios of at least one generator, which justifies the use of the scenarios pre-selection within the third filter. One can observe from Table III that this prefilter helps selecting a reasonable number of scenarios (subset $\left|\mathcal{S}_{s}^{3}\right|$ ) from the set of postulated scenarios. Best performance of this pre-filter appears for the larger 1203-bus system, where only 46 scenarios (out of 876) are pre-selected. Furthermore, Table IV shows that the use of pre-selection leads to a better ranking of binding scenarios according to $S I_{3}$, especially low ranked binding scenarios climbing significantly in the ranking (e.g. thanks to this pre-filter, in the 618-bus system, scenario 677 climbs from place 42 to 18 ). This pre-filter also has the ability to correctly filter out top ranked scenarios, which reactive reserves requirements are covered by other scenarios, e.g. as is the case of two top 7 scenarios (the second and the sixth) in the 618-bus system. The limit of this pre-filter performance is that the number of binding scenarios may be at most equal ${ }^{11}$ with the number of generators.

2) Third filter ranking performance: Table IV highlights the very good filtering ability of the third filter since most binding scenarios are top ranked, e.g. for the Nordic32 system and the 1203-bus system, 5 (out of 6) binding contingencies

\footnotetext{
${ }^{11}$ Assuming that each binding scenario constraints a different generator reactive power reserve.
}

are ranked in the top 5. On the other hand, scenarios with smaller but very local overall reactive power response (e.g. scenarios 4, 677, 388 in respectively the systems Nordic32, 618-bus, and 1203-bus) are not identified by the filter at the first iteration.

3) First filter ranking performance: Clearly, as long as the SCOPF does not include all binding scenarios at the optimum, the current optimal reactive reserves are insufficient to ensure a (viable) steady-state for all scenarios. Table III shows that at the second iteration a significant number of scenarios (subset $\left|\mathcal{S}_{s}^{1}\right|$ ) do not have a power flow solution (e.g. 27 scenarios for the 618-bus system) which justifies the use of the PFOM technique to rank them, since a classical power flow program diverges leaving us without any information. Table IV shows that the $S I_{1}$-based filter ranks to the top the remaining binding scenarios at the optimum and hence allows to accurately identify them (e.g. for the 618-bus system the binding scenarios 514, 680, and 677 are ranked in top 4 among the 27 scenarios, for the IEEE118 system the binding scenarios 125 and 147 are ranked in top 3 among the 14 scenario, etc.).

4) CPU times: The last column of Table III yields the CPU times elapsed, on a PC with 1.9-GHz and 2-Gb RAM, in each iteration of the algorithm. The three filters are extremely fast since they combine the quantities provided by the SA (e.g. they take together $0.01 \mathrm{~s}$ in the largest system). The average elapsed time for solving the SCOPF program including 5 contingencies is of: $0.8 \mathrm{~s}, 4.2 \mathrm{~s}, 13.1 \mathrm{~s}$, and $42.5 \mathrm{~s}$ for the Nordic32 system, IEEE118 system, 618-bus system, and 1203-bus system. The average elapsed time in the PFOM program to compute the load flow solution for a scenario is of: $0.01 \mathrm{~s}, 0.02 \mathrm{~s}, 0.06 \mathrm{~s}$, and 0.15 s for the Nordic 32 system, IEEE118 system, 618 -bus system, and 1203-bus system. Note that these CPU times do not consider parallel computations, SCOPF warm/hot start, and the SCOPF code was not focused on optimizing computational speed. Nevertheless, since the ancillary service of reactive reserves assessment is performed off-line, the computational speed not being hence a big concern, the obtained execution times indicate that the proposed approach may be applied in a reasonable amount of time in large systems.

\section{E. Observations concerning the MONRPR SCOPF solution}

Extensive simulations with the approach have led to the following observations concerning the SCOPF optimal solution:

- Withdrawing a small amount of the optimal needed reserve of any generator leads, for at least one (binding) scenario, to the lack of a power flow solution and/or violated operating limits. In other words the loadability margin with respect to any binding scenario is zero.

- For binding scenarios constrained by voltage stability for at least one generator both constraints (7) and (8) are binding (i.e. $Q_{g i}^{s}=Q_{g i}^{0}+R_{g i}^{O C \star}$ and $V_{g i}^{s}=V_{g i}^{i m p}$ ) signifying that the system is at a breaking point [3]. No loadability limit of type saddle node bifurcation have been encountered. This is however an expected outcome given the problem objective, since the higher the generators voltage the less the needed reserves, and hence the voltage of some generators under reactive power limit tends to its maximum allowed value $V_{g i}^{i m p}$. 


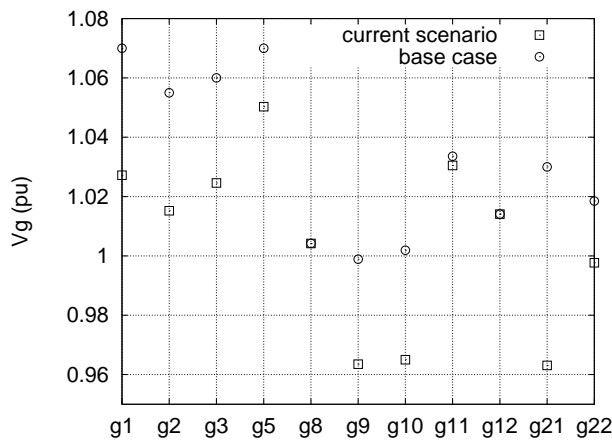

Fig. 6. Nordic32 system: voltage of generators that reached their reactive power limit in a binding scenario (loss of line 4071-4072).

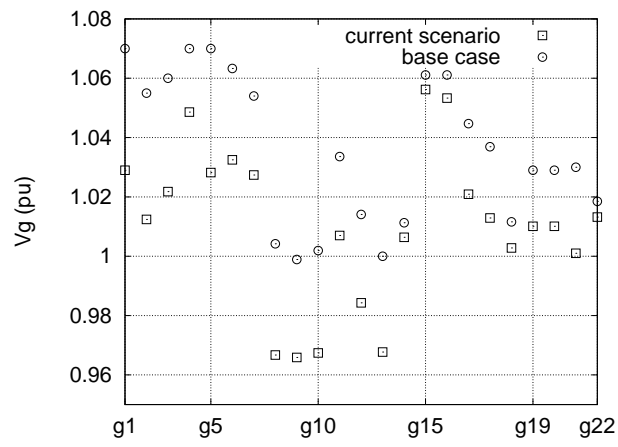

Fig. 7. Nordic32 system: voltage of all generators in a non-binding scenario (loss of line 4042-4043).

- The generator mode under voltage control (i.e. if $Q_{g i}^{s}<$ $Q_{g i}^{0}+R_{g i}^{O C \star}$ then $V_{g i}^{s}$ tends to $V_{g i}^{i m p}$ ), which is not modeled by the weak constraints (7) and (8), is always satisfied for the binding scenarios at the SCOPF solution. Fig. 6 provides the voltages at the SCOPF solution for the generators that have reached their limit in a binding scenario. Observe that for two generators ( $g 8$ and g12) both constraints are active: $V_{g i}^{s}=V_{g i}^{i m p}$ and $Q_{g i}^{s}=Q_{g i}^{0}+R_{g i}^{O C \star}$, while for other limited generators the terminal voltage has dropped with respect to $V_{g i}^{i m p}$.

- Obviously, the generator mode under voltage control is not met for the non-binding scenarios, where since $V_{g i}^{s}$ does not have any impact on the objective value, it can converge to any value between its limits (15) provided that voltage bounds at all buses (14) are satisfied. Fig. 7 provides the voltage at the SCOPF solution for all generators in a non-binding scenario. Observe that, although no generator reached its reactive power limit (i.e. $\left.Q_{g i}^{s}<Q_{g i}^{0}+R_{g i}^{O C \star}\right)$ their voltages are lower than $V_{g i}^{i m p}$.

In order to confirm that the SCOPF optimum corresponds indeed to a breaking point the dynamic simulation, described in the next Section, is used. Fig. 8 shows the evolution of the sensitivity $\mathrm{dQgt} / \mathrm{dQ}$ l of the total generated reactive power with respect to the reactive power at a bus for two scenarios which are voltage unstable for the SCOPF-based reserves. These sensitivities change sign when the system passes through the so-called critical point which initiates voltage instability [3]. Note that since in both cases the change in sign is caused

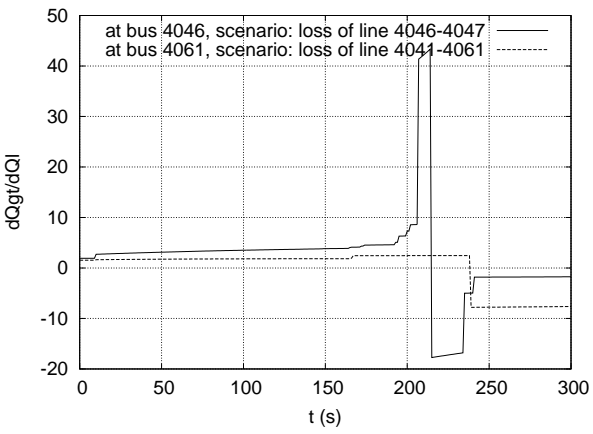

Fig. 8. Evolution of dQgt/dQ1 sensitivity in two voltage unstable scenarios.

by a generator switching under reactive power limit (i.e. g7 at $t=215 \mathrm{~s}$ in scenario $4046-4047$ and g18 at $t=239 \mathrm{~s}$ in scenario 4041-4061) then the critical point is indeed a breaking point. As a consequence, one can observe that the largest (positive) value of the sensitivities is reasonably small (especially for scenario 4041-4061) .

\section{F. Additional reserves to ensure long-term voltage stability}

The procedure proposed in Section III is illustrated for the Nordic32 system.

1) Dynamic simulation assumptions: The long-term system response to each scenario has been obtained by Quasi SteadyState Simulation (QSSS) [3]. The long-term dynamics are driven by load restoration mechanisms and Over-Excitation Limiters (OELs), both acting with various delays. Voltage instability is declared if the system passes through the critical point or short-term equilibrium is lost [3].

Two load models are used in QSSS: exponential and restorative. When an exponential load model is used in QSSS, all 22 loads (in SCOPF loads are modeled as constant power at the transmission level) are represented behind their distribution transformers, all equipped with Load Tap Changers (LTCs).

The exponential load model is described by the equations:

$$
P_{l i}^{s}=z_{P_{l i}} P_{l i}^{0}\left(V_{l i}^{s} / V_{l i}^{o}\right)^{\alpha_{t}}, \quad Q_{l i}^{s}=z_{Q_{l i}} Q_{l i}^{0}\left(V_{l i}^{s} / V_{l i}^{o}\right)^{\beta_{t}},
$$

where $z$ is the dimesionless load demand, $\alpha_{t}=1$ and $\beta_{t}=2$.

The restorative load model is described by the equations:

$$
\begin{aligned}
T_{P} \dot{z}_{P_{l i}} & =\left(V_{l i}^{s} / V_{l i}^{o}\right)^{\alpha_{l}}-z_{P_{l i}}\left(V_{l i}^{s} / V_{l i}^{o}\right)^{\alpha_{t}} \\
T_{Q} \dot{z}_{Q_{l i}} & =\left(V_{l i}^{s} / V_{l i}^{o}\right)^{\beta_{l}}-z_{Q_{l i}}\left(V_{l i}^{s} / V_{l i}^{o}\right)^{\beta_{t}},
\end{aligned}
$$

with $\alpha_{l}=\beta_{l}=0$ (forcing the load to restore to its base case value), $\alpha_{t}=1, \beta_{t}=2$, and time constant $T_{P}=T_{Q}=30 \mathrm{~s}$.

2) Results with exponential and restorative load models: Figure 9 shows the evolution of the total system load for the most severe scenario 4046-4047, using both load models. Observe that, when reserves are provided by SCOPF, for the restorative model the system is voltage unstable (the minimum unrestored load is around $20 \mathrm{MW}$ ), while for the exponential model the system is voltage stable. This is due to the deadband effect of LTCs transformers, the final total load being $50 \mathrm{MW}$ lower than in the base case. The figure also pinpoints that, if appropriate additional reserves are available, for the restorative model the total load coincides to its base case value. 


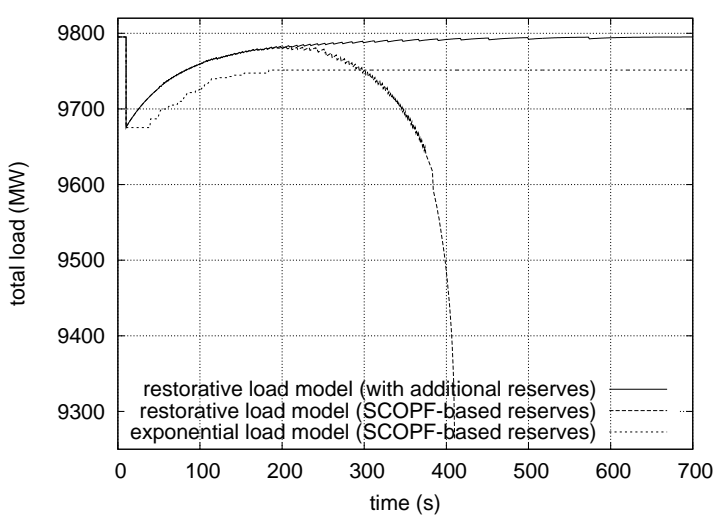

Fig. 9. Total load evolution using two load models for the scenario: loss of line 4046-4047.

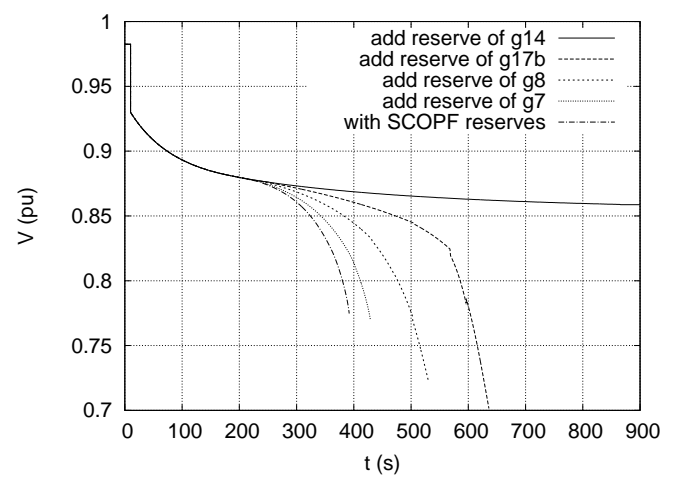

Fig. 10. Voltage evolution at bus 4046 for successive added reserves.

Note that, when using the exponential load model, the most widely used for long-term voltage stability studies, all scenarios are found voltage stable for the reactive reserves provided by the SCOPF. This observation confirms the general belief that SCOPF constant power load model is often conservative with respect to long-term voltage stability driven by LTCs and OELs dynamics. On the other hand with the restorative load model 4 scenarios are unstable (see Table V).

In order to illustrate the proposed approach only the restorative load model is considered hereafter.

3) Additional reactive reserves to ensure the voltage stability of a scenario: Let us consider the loss of line 4046-4047.

Fig. 10 plots the voltage evolution at bus 4046, where the voltage drops the most, for the reserves provided by SCOPF and by allowing sequentially the whole physical reserve of the most effective generator ${ }^{12}$. As more reserve is available on proper generators the system collapses later and eventually becomes voltage stable. In order to stabilize this scenario appropriate additional reserves are required at least on the four most effective generators.

Fig. 11 indicates that the overall additional reactive reserve decreases nonlinearly as the number of effective generators

\footnotetext{
${ }^{12}$ The most effective generator has the largest value of the sensitivity of the voltage which falls the most with respect to the generator reactive power injection, computed at the critical point of the voltage unstable trajectory [26]. This sensitivity is non-zero only for generators operating on over-excitation mode at the critical point.
}

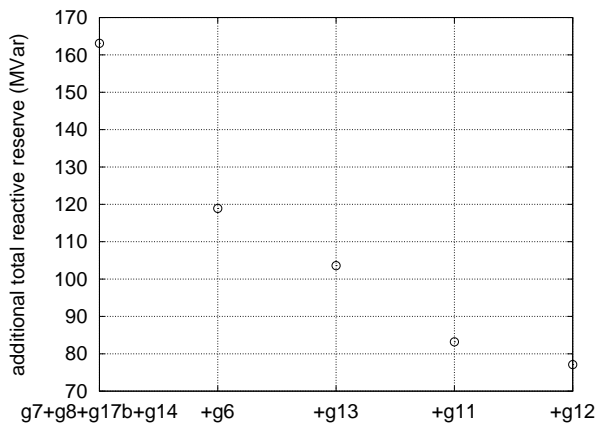

Fig. 11. Overall needed reactive power reserve vs. number of effective generators for which the whole physical reserve is available.

TABLE V

INDIVIDUAL ADDITIONAL RESERVES (MVAR) FOR VOLTAGE UNSTABLE SCENARIOS AND OVERALL SOLUTION OF THE PROPOSED APPROACH

\begin{tabular}{|c|c|c|c|c|c|}
\hline \multirow[t]{2}{*}{ gen } & \multicolumn{4}{|c|}{ scenario: loss of line } & \multirow{2}{*}{$\begin{array}{c}\text { overall } \\
\text { solution }\end{array}$} \\
\hline & $4046-4047$ & | 4043-4047 & 4041-4061 & 1021-1022 & \\
\hline g7 & 8.1 & 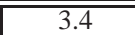 & & & 8.1 \\
\hline g8 & 7.3 & 5.8 & & & 7.3 \\
\hline g17b & 16.7 & 8.3 & & & 16.7 \\
\hline g14 & 15.4 & 3.0 & & & 15.4 \\
\hline g6 & 6.5 & 3.4 & & & 6.5 \\
\hline g13 & 6.8 & 1.6 & & & 6.8 \\
\hline g11 & 7.7 & 3.0 & & & 7.7 \\
\hline g12 & 8.6 & & & & 8.6 \\
\hline $\mathrm{g} 15$ & & 44.5 & & & 14.3 \\
\hline g19 & & & 4.5 & & 4.5 \\
\hline g4 & & & & 13.3 & 13.3 \\
\hline total & 77.1 & 70.0 & 4.5 & 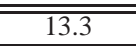 & 110.2 \\
\hline
\end{tabular}

increases. The minimum reserve is obtained when the eight generators, that enter on over-excitation mode whatever the reserves of other generators, maintain adequate reserves.

As for the SCOPF, the minimum overall reserve for a scenario coincides with the generators response to the scenario assuming their whole physical reserves are available.

4) Additional reactive reserves to ensure the voltage stability of several scenarios: Table $\mathrm{V}$ provides the individual additional reserves for each voltage unstable scenario and the overall solution provided by the proposed procedure.

When applying the proposed procedure the reserve requirement of scenario 4046-4047 are first satisfied. However, at the next step, only scenario 4043-4047 benefits of these larger reserves, generator g15 requiring 44.5-14.3 = 30.2 MVar less. This is due to, unless scenarios 4046-4047 and 4043-4047, other scenarios impact far away areas and hence do not have common generators that enter in over-excitation mode, needing thereby additional reserves to ensure voltage stability.

Note that at the overall solution each generator intervenes with its maximum required reserve over all individual scenarios, unless for generator g15 where the overall solution gains 30.2 MVar with respect to the overlap of individual solutions.

This procedure provides optimal additional reserves to ensure voltage stability only if unstable scenarios need larger reserves on different generators and/or on some common generators provided that the reserves required by the worst scenario among them satisfy also the other scenarios.

As regards the execution time QSSS proved its compatibility 
with real-time requirements for reasonably large systems [3].

\section{CONClusions}

This paper has proposed a novel approach to evaluate generators reactive power reserves with respect to a set of postulated operating scenarios. The main features of this approach are:

- it combines sequentially SCOPF and dynamic simulation providing reserves that satisfy both operating constraints (limits on voltages and currents) and voltage stability, as opposite to most existing methods which focus only on voltage stability and adopt static models;

- the optimal values of reserves with respect to operating constraints are computed using a transparent systemwide performance criterion namely the minimum overall needed reactive reserve, as opposite to most existing methods whose output depends on the choice of the weights assigned to individual reserves;

- the large size of the SCOPF is considerably reduced to more tractable problems by using appropriate techniques to filter-out non-binding scenarios;

- it uses simple constraints to model the switch between generator operation modes which satisfy the standard modeling by complementarity constraints but avoid the reliability problems posed by the latter;

- as all existing methods, the optimality of the additional reserves to ensure voltage stability cannot be guaranteed. The proposed procedure provides optimal additional reserves only in particular cases.

The method can be used in operational planning to assess the reactive power reserves of the forecasted operating points, can be easily integrated within a reactive reserves market, and can be straightforwardly extended to the reactive power absorption mode.

\section{ACKNOWLEDGMENTS}

I would like to thank Prof. Thierry Van Cutsem (ULg) for the fruitful discussions during my $\mathrm{PhD}$ studies about the evaluation of reactive power reserves and for giving me access to QSSS, and Prof. Costas Vournas (NTUA) for the suggestion concerning the modeling of generators switch between different operation modes in the context of loadability margin computation by OPF.

I thank RTE (the French TSO) for allowing me to use and publish results with their data.

\section{REFERENCES}

[1] P. Kundur, J. Paserba, V. Ajjarapu, G. Andersson, A. Bose, C. Canizares, N. Hatziargyriou, D. Hill, A. Stankovic, C. Taylor, T. Van Cutsem, and V. Vittal, "Definition and classification of power system stability", IEEE Trans. on Power Syst., Vol. 19, No. 4, 2004, pp. 1387-1401.

[2] C. W. Taylor, Power System Voltage Stability, McGraw Hill, EPRI Power System Engineering Series, 1994.

[3] T. Van Cutsem and C. Vournas, Voltage Stability of Electric Power Systems, Kluwer Academic Publisher, 1998.

[4] E. Khan, and R. Baldick, "Reactive Power is a Cheap Constraint", The Energy Journal, Vol. 15, No. 4, 1994, pp. 191-201.

[5] S. Hao and A. Papalexopoulos, "Reactive power pricing and management", IEEE Trans. on Power Syst., Vol. 12, No. 1, 1997, pp. 95-104.

[6] J. W. Lamont and J .Fu, "Cost analysis of reactive power support", IEEE Trans. on Power Syst., Vol. 14, No. 3, 1999, pp. 890-898.
[7] K. Bhattacharya and J. Zhong, "Reactive power as an ancillary service", IEEE Trans. on Power Syst., Vol. 16, No. 2 , 2001, pp. 294-300.

[8] G. Gross, S. Tao, E. Bompard and G. Chicco, "Unbundled Reactive Support Service: Key Characteristics and Dominant Cost Component", IEEE Trans. on Power Syst., Vol. 17, No. 2, 2002, pp. 283-289.

[9] I. El-Samahy, K. Bhattacharya, C. Canizares, M. Anjos, and J. Pan, "A Procurement Market Model for Reactive Power Services Considering System Security", IEEE Trans. on Power Syst., Vol. 23, No. 1, 2008, pp. 137-149.

[10] W. Xu, Y. Zhang, L.C.P. da Silva, P. Kundur, and A. Warrack, "Valuation of dynamic reactive power support services for transmission access", IEEE Trans. on Power Syst., Vol. 16, No. 4, 2001, pp. 719-728.

[11] B. Avramovic and L. H. Fink, "Real-time Reactive Security Monitoring", IEEE Trans. on Power Syst., Vol. 7, No. 1, 1992, pp. 432-437.

[12] R. A. Schlueter, "A Voltage Stability Security Assessment Method", IEEE Trans. on Power Syst., Vol. 13, No. 4, 1998, pp. 1423-1438.

[13] C.W. Taylor and R. Ramanathan, "BPA Reactive Power Monitoring and Control following the August 10, 1996 Power Failure", SEPOPE conference, Salvador (Brazil), May 1998, paper IP-003.

[14] F. Capitanescu and T. Van Cutsem, "Evaluation of reactive power reserves with respect to contingencies", IREP conference, Onomichi (Japan), 2001, pp. 377-386.

[15] D. Dong, B. Chowdhury, M. Crow, and L. Acar, "Improving voltage stability by reactive power reserve management", IEEE Trans. on Power Syst., Vol. 20, No. 1, 2005, pp. 338-345.

[16] H. Song, B. Lee, S.-H. Kwon, and V. Ajjarapu, "Reactive reserve-based contingency constrained optimal power flow (RCCOPF) for enhancement of voltage stability margins", IEEE Trans. on Power Syst., Vol. 18, No. 4, 2003, pp. 1538-1546.

[17] B. Leonardi and V. Ajjarapu, "Investigation of various generator reactive power reserve (GRPR) definitions for online voltage stability/security assessment", IEEE PES general meeting, Pittsburgh (USA), 2008.

[18] P. A. Ruiz and P. Sauer, "Reactive power reserve issues", NAPS conference, Carbondale (USA), 2006.

[19] D. Pudjianto, G. Strbac, S. Ahmed, K. Bell, and P. Turner, "Value of VAR support in a competitive environment", PSCC conference, Seville (Spain), June 2002.

[20] W. Rosehart, C. Roman, and A. Schellenberg, "Optimal power flow with complementarity constraints", IEEE Trans. on Power Syst., Vol. 20, No. 2, 2005, pp. 813-822.

[21] L. Platbrood, H. Crisciu, C. Merckx, S. Fliscounakis, P. Panciatici, F. Capitanescu, L. Wehenkel, M. Ortega-Vazquez, and D. Kirschen, "Deliverable D3.3: Prototypes for European transmission system steadystate optimisation", PEGASE project, http://www.fp7-pegase.eu/, 2011.

[22] F. Capitanescu, M. Glavic, D. Ernst, and L. Wehenkel, "Contingency filtering techniques for preventive security-constrained optimal power flow", IEEE Trans. on Power Syst., Vol. 22, No. 4, 2007, pp. 1690-1697.

[23] A. Sasson, C. Trevino, F. Aboytes, "Improved Newton's load flow through a minimization technique", IEEE Trans. on PAS, Vol. PAS-90, 1971, pp. 1974-1981.

[24] F. Capitanescu, "Preventive assessment and enhancement of power system voltage stability: an integrated approach of voltage and thermal security", PhD thesis, University of Liège, 2003, available on-line at: http://orbi.ulg.ac.be/handle/2268/28220.

[25] B. Stott, O. Alsac, and A.J. Monticelli, "Security analysis and optimization" (Invited Paper), IEEE Proc., Vol. 75, No. 12, 1987, pp. 1623-1644.

[26] F. Capitanescu and T. Van Cutsem, "Unified sensitivity analysis of unstable or low voltages caused by load increases or contingencies", IEEE Trans. on Power Syst., Vol. 20, No. 1, 2005, pp. 321-329.

[27] F. Capitanescu, M. Glavic, D. Ernst, and L. Wehenkel, "Interior-point based algorithms for the solution of optimal power flow problems", Electric Power Syst. Research, vol. 77, no. 5-6, April 2007, pp. 508-517.

[28] CIGRE Task Force 38.02.08, "Long-Term Dynamics, Phase II", 1995.

[29] IEEE118 bus system, available online at http://www.ee.washington.edu, 1996.

Florin Capitanescu was born in Romania on May 16, 1973. He graduated in Electrical Power Engineering from the University "Politehnica" of Bucharest in 1997. He obtained the Ph.D. degree from the University of Liège in 2003. His main research interests include optimization methods, in particular optimal power flow, and voltage stability. 